\title{
VERHALTENSTHERAPIE - Mit dem ersten Dezennium ins neue Millennium
}

Mit dem neuen Millennium geht VERHALTENSTHERAPIE in das letzte Jahr ihres ersten Dezenniums. Im «Gründungs-Editoral» (Heft 1, 1991) haben Uli Wittchen und ich die wesentlichen Zielsetzungen der Zeitschrift und aus unserer subjektiven Sicht wichtige, der Klärung harrende inhaltliche, berufs- und gesundheitspolitische Fragen zur Verhaltenstherapie dargestellt. Ein Jahr später stellten wir die rückblickend - sehr voreilige Frage: «VERHALTENSTHERAPIE - Aufgaben, Ziele und Erwartungen erreicht?» Jetzt, am Beginn des ersten Jahrzehnts unserer Zeitschrift, soll diese Frage so nicht wiederholt werden. Stattdessen einige persönliche Anmerkungen zu unterschiedlichen Entwicklungen, die teils Genugtuung und Freude ausgelöst haben, teils die Neugierde aufrechterhalten, teils aber auch Sorgen bereiten:

Die Gruppe der aktiven Mitgestalter der Zeitschrift konnte deutlich vergrößert werden. Seit 1997 hat sich die Hauptschriftleitung erweitert und leicht verändert. Im kommenden Jahr wird sich dieser Prozess fortsetzen, und auch im Editorial Board fand und findet kontinuierlich ein Veränderungsprozess statt. Ferner konnten zunehmend engagierte Gastherausgeber für spezielle Themenhefte gewonnen werden. Der Manuskripteingang wurde auf diese Weise deutlich erhöht.

Unsere Zeitschrift ist als Forschungs- und Informationsforum mit einem Impakt-Faktor von etwa 0,5 (1998) recht gut platziert. Hier können wir mit Hilfe unserer Autorinnen und Autoren aber sicher noch deutlich zulegen. In diesem Zusammenhang seien alle Autorinnen und Autoren nochmals herzlich gebeten, ihre Zusammenfassungen umfangreicher und strukturiert zu gestalten (Einzelheiten in den Autorenrichtlinien). Denken Sie daran, dass das englische Summary jedes Artikels in vielen Literaturdatenbanken und im Internet erscheint. Eine aussagekräftige Zusammenfassung erleichtert somit anderen Autoren das Auffinden (und Zitieren) Ihrer Arbeit.

Unsere Zeitschrift ist inzwischen «im Markt fest etabliert» und wirtschaftlich gesund, obwohl sie nahezu werbungsfrei ist -letzteres ein durchaus veränderungswürdiger Zustand. Sie ist zum Mitteilungsorgan für zahlreiche verhaltenstherapeutische Weiterbildungseinrichtungen und Verbände geworden.

Die Anzahl der kassenzugelassenen Verhaltenstherapeuten stieg von 2247 (1632 Psychologen und 615 Ärzte) im Grün- dungsjahr der Zeitschrift, 1991, bis Ende 1998 auf 4900 an (3736 Psychologen und 1164 Ärzte). Die tiefenpsychologischen und analytischen Therapeuten (inklusive der Kinderund Jugendtherapeuten) überwiegen jedoch mit 12832 (8571 Ärzte, 4261 Psychologen) immer noch deutlich. Insgesamt waren also im letzten Jahr vor dem Psychotherapeutengesetz (PTG) 16168 Psychotherapeuten kassenzugelassen. Bis Ende 1999 reduzierte sich diese Zahl im Rahmen der Umsetzung des PTG auf 15800 (davon 11530 Psychologen und 4270 Ärzte; die Aufteilung nach den beiden Therapierichtungen lag bei Redaktionsschluss noch nicht vor).

Die Ursachen für den allgemeinen (in einigen Bundesländern dramatischen) Abfall des Punktewertes für die Psychotherapieleistungen sind noch nicht geklärt: Mehr Leistungen durch weniger Therapeuten? Vorenthaltung von Teilbeträgen aus dem früheren Erstattungsverfahren durch die Krankenversicherungen? Weiterhin hohe Kosten im weiterbestehenden Erstattungsverfahren? Hier liegt gegenwärtig eines der größten Gefährdungsmomente für die Konsolidierung des psychotherapeutischen Versorgungssystems, die mit dem PTG eigentlich erwartet wurde.

Die Qualitätssicherung der Verhaltenstherapie wurde in Teilbereichen ebenfalls gefährdet. Die Weiterbildungsordnung für den relativ neuen «Facharzt für Psychiatrie/Psychotherapie» ist dringend veränderungsbedürftig, da die Bundesrahmenrichtlinien die Qualität der Verhaltenstherapie in diesem Curriculum nicht hinreichend sichern. Erfreulicherweise sind Nachbesserungen in Vorbereitung. Die Qualität der Verhaltenstherapie durch psychologische Therapeuten ist durch die Übergangsregelungen nach dem PTG ebenfalls teilweise gefährdet worden: Auf der einen Seite wurden Therapeuten aus dem Erstattungsverfahren zur «Besitzstandswahrung» auch dann zugelassen, wenn nur eine verhaltenstherapeutische Teilqualifizierung vorlag, andererseits wurden aber vollqualifizierte Verhaltenstherapeuten durch das «Zeitfenster» aus dem System ausgeschlossen. Auch hier sind über einige Jahre Qualitätssicherungsmaßnahmen unbedingt erforderlich. Das Problem ist jedoch in beiden Berufsgruppen grundsätzlich lösbar.

Mit dem PTG hat auch die Diskussion um das «GutachtenVerfahren» in der Richtlinien-Psychotherapie erneut an Bri- 
sanz gewonnen. Es gibt viele interessante Vorschläge - von unterschiedlichen Modifikationen des Verfahrens bis hin zu dessen völliger Auflösung. Der Dialog zwischen Therapeuten und Gutachtern läuft im Verfahren teils exzellent, teils katastrophal, überwiegend aber reibungslos (wenn auch oft mit «schweigsamem Frust»). Der qualitätssichernde Nutzen ist umstritten. Außerhalb des Verfahrens könnten der Dialog und die Zufriedenheit miteinander durch gemeinsame Workshops sicher wesentlich verbessert werden; zusammen mit einer adäquat angehobenen Honorierung der Berichtserstattung wäre vielleicht sogar eine freudvollere Akzeptanz zu erzielen.

Von besonderem Interesse wird auch die weitere Entwicklung der naturgemäß unvermeidbaren Endlosdiskussionen um die essentiellen Inhalte der VT und um ihre adäquate Benennung sein (Verhaltenstherapie alleine oder in Kombination mit: kognitiv, emotional, kognitiv-emotional, multi-modal, strategisch-systemisch u.ä.). Auch die Diskussion um das vereinheitlichende Grundkonzept für alle Psychotherapien oder aber um die «Neue Psychotherapie» - als Resultat aus allen hilfreichen Erkenntnissen unterschiedlicher Psychotherapierichtungen - wird unvermindert und anregend fortgesetzt. Bei aller Begeisterung für unsere eigene Kreativität müssen wir aber mehr denn je die Patienten (oder «Kunden», wie man heute sagt) und deren Krankenversicherungen als Empfänger unserer Botschaften im Auge haben, d.h., diese müssen verständlich, klar und akzeptabel sein - und das resultierende Behandlungsangebot muss ein wirksamer Kompromiss zwischen Ideal und Finanzierbarkeit werden.

In diesem Sinne, wie schon 1991, auch weiterhin die persönliche Hoffnung: «Verhaltenstherapie» steht für eine dynamische und flexible Therapierichtung, die sich durch immer neue kreative klinische Innovationen und deren begleitende «rigide» Beforschung auszeichnet. Bitte nutzen Sie die Zeitschrift VERHALTENSTHERAPIE auch weiterhin intensiv für diesen Prozess.

\section{Hand, Hamburg}

\section{Dank an die Gutachter}

Die Hauptschriftleitung der Zeitschrift VERHALTENSTHERAPIE dankt sehr herzlich allen Gutachtern, die im Jahre 1999 durch ihre Reviews zur Qualität der Zeitschrift in hohem Maße beigetragen haben:

G. Buchkremer, Tübingen

B. Dahme, Hamburg

A. Ehlers, Oxford (UK)

P. Fiedler, Heidelberg

R. Frank, Gießen

W. Gerber, Kiel

F. Gerhards, Trier

K. Hahlweg, Braunschweig

W. Hauke, Windach

M. Hautzinger, Tübingen

F. Hohagen, Lübeck

R. de Jong-Meyer, Münster

G. Kockott, München

M. Linden, Berlin

A. Maercker, Dresden

R. Meermann, Bad Pyrmont

N. Münchau, Hamburg

D. Nutzinger, Bad Bramstedt

H. Peter, Hamburg

H. Reinecker, Bamberg

E.-R. Rey, Mannheim

H. Rüddel, Bad Kreuznach

G. Schmidt, Hamburg

S. Sulz, München

D. Vaitl, Gießen

M. Zaudig, Windach 